\title{
Fuentes de conocimiento en la identificación y preferencias de fauna en niñez de contextos rurales y urbanos
}

\author{
Sources of Knowledge in Preferences and Identification of Fauna in Children of Rural and Urban Contexts \\ Fontes de conhecimento na idade da infância, na identificação e preferências da fauna, nos \\ contextos rurais e urbanos
}

Nidia Yaneth Torres-Merchan ${ }^{1}$

Universidad Pedagógica y Tecnológica de Colombia

Tunja, Colombia

nidia.torres@uptc.edu.co

(iD http://orcid.org/0000-0003-4813-6428

Luis Alfonso Salcedo-Plazas ${ }^{2}$

atpgin

Universidad Pedagógica y Tecnológica de Colombia

Tunja, Colombia

luis.salcedo@uptc.edu.co

(iD http://orcid.org/0000-0001-6246-014x

Angela Becerra-Niño3

Universidad Pedagógica y Tecnológica de Colombia

Tunja, Colombia

becerras324@gmail.com

Wilson Valderrama ${ }^{4}$

Universidad Pedagógica y Tecnológica de Colombia

Tunja, Colombia

wilsonvalde@gmail.com

Recibido • Received $\cdot$ Recebido: 17 / 11 / 2016
Corregido • Revised • Revisado: 03 / 04 / 2018
Aceptado • Accepted • Aprovado: 05/ 06 / 2018

\footnotetext{
${ }^{1}$ Licenciada en Biología y Química por la Universidad Pedagógica y Tecnológica de Colombia. Magister en Docencia de la Química de la Universidad Pedagógica Nacional y Doctora en Didácticas Específicas Ciencias A partir del año 2010, trabaja como docente de planta en la Universidad Pedagógica y Tecnológica de Colombia en el programa de Licenciatura en Ciencias Naturales y Educación Ambiental, que se centra en la preparación de profesorado de ciencias para el nivel de educación básica. Ha desarrollado investigaciones en dos líneas básicas: Cuestiones socio-científicas y pensamiento crítico. Pertenece al grupo de investigación WAIRA escalonado y reconocido por COLCIENCIAS en Colombia. En este grupo trabaja en la línea de investigación innovación e investigación en didáctica de las ciencias.

${ }^{2}$ Licenciado en Matemáticas y Física con especialización en Estadística y candidato a magister en la misma área. Trabaja como docente de planta en la Universidad Pedagógica y Tecnológica de Colombia en el programa de Matemáticas, del cual ha sido director. Ha publicado en revistas como Ciencia en Desarrollo; Salud Historia y Sanidad. Pertenece al grupo de investigación GAMMA, avalado por la Universidad Pedagógica y Tecnológica de Colombia.

${ }^{3}$ Licenciada en Ciencias Naturales y Educación Ambiental, Semillero de investigación grupo WAIRA Universidad Pedagógica y Tecnológica de Colombia.
}

${ }^{4}$ Licenciado en Ciencias Naturales y Educación Ambiental, Semillero de investigación grupo WAIRA Universidad Pedagógica y Tecnológica de Colombia. 
doi: http://dx.doi.org/10.15359/ree.22-3.4

URL: http://www.una.ac.cr/educare

CORREO: educare@una.cr

Resumen: Este artículo analiza la influencia de los medios de comunicación en el conocimiento de algunas especies de animales en estudiantes de educación primaria, por tanto, se valoran las percepciones que trasmiten las imágenes sobre grupos taxonómicos de fauna en 249 niños y niñas de básica primaria de contextos rurales y urbanos con edades de 7 a 13 años. Para ello, se utilizaron dos presentaciones en PowerPoint, en las que se muestran imágenes de especies endémicas y no endémicas, de los siguientes grupos taxonómicos: reptiles, anfibios, aves y artrópodos. A partir de estas se diseñaron matrices con diferentes opciones de respuesta para registro de la información de acuerdo con dos preguntas: ¿Dónde ha visto el animal? ¿Lo considera agradable o desagrabable? Se encuentra que, en el escenario urbano y rural, la televisión y el internet son los medios que permiten mayor identificación de las especies presentadas. En relación con las percepciones del estudiantado, se muestra mayor agrado por especies de mamíferos y aves. Estos datos permiten inferir la influencia que pueden ejercer los medios de comunicación en el conocimiento de la biodiversidad y el fomento del cuidado hacia la naturaleza.

Palabras claves: Conservación; carismático; no carismático; medios de comunicación; educación.

Abstract: This article analyzes the influence of mass media on primary school students when studying animal species. Images on taxonomic groups of wildlife transmit perceptions. Therefore, these transmitted perceptions are examined in 249 children from rural and urban elementary school aged 7 to 13 years old. This analysis used two PowerPoint presentations with images of endemic and non-endemic species. It is found that in urban and rural contexts, television and Internet are the means for further identification of the species presented. Concerning the perceptions, students have a preference for mammals and birds species. These data allow inferring the influence media can have on the knowledge of biodiversity and the promotion of care towards nature.

Keywords: Conservation; charismatic; not charismatic; media; education.

Resumo: Este artigo analisa a influência dos meios de comunicação no conhecimento das crianças do ensino fundamental sobre algumas espécies animais. Portanto, valorizam-se as percepções transmitidas pelas imagens sobre grupos taxonômicos da fauna em 249 crianças do ensino fundamental, de contextos rural e urbano, com idades de 7 a 13 anos. Para isso, se utilizaram duas apresentações PowerPoint onde são mostradas imagens de espécies endêmicas e não endêmicas, dos seguintes grupos taxonômicos: répteis, anfíbios, aves e artrópodes. A partir dessas, desenharamse matrizes com diferentes opções de resposta para o registro das informações de acordo com duas questões: Onde o animal foi visto? Você considera isso agradável ou desagradável? Como resultado, no cenário urbano e rural, a televisão e a internet são os meios que permitem maior identificação das espécies apresentadas. Em relação com as percepções dos estudantes, indicam maior agrado para espécies de mamíferos e aves. Estes dados permitem inferir a influência que podem exercer os meios de comunicação sobre o conhecimento da biodiversidade e a promoção do cuidado com a natureza.

Palavras-chave: Conservação; carismático, não carismático; meios de comunicação; educação. 


\section{Introducción}

Estudios (Braund, 1998; Lindemann-Matthies, 2006; Pooley y O'connor, 2000; Tarlowski, 2006) han encontrado que los efectos de las experiencias directas con la naturaleza en niñez rural y urbana influyen directamente sobre su preferencia hacia la biodiversidad. Sin embargo, es cierto que parte de esas preferencias e identificación de las especies son producto, entre otras, de campañas publicitarias lideradas por diversas ONG, que en su mayoría están enfocadas a especies carismáticas; es el caso del pingüino de ojos amarillos Megadyptes en Nueva Zelanda (Tisdell, 2011). El mismo autor, señala que, aunque muchas de las ONG combinan esfuerzos por su conservación; otras, por el contrario, no ejercen influencia directa sobre los bienes ambientales, sino simplemente actúan como grupos de presión, que tratando de influir en la política pública.

En relación con las preferencias sobre especies de fauna, trabajos de Morse-Jones et al. (2012) muestran que habitantes del Reino Unido están en disposición de aportar significativas sumas de dinero, para conservar especies carismáticas o endémicas. Esto mismo sucede con respecto a la conservación de especies silvestres en las montañas de Tanzania; en contraste, con especies que no son ni endémicas ni carismáticas. Algo similar fue reportado en los estudios de Horton, Colarullo, Bateman y Peres (2003), quienes señalan que personas residentes en Italia y el Reino Unido están dispuestas a dar donativos para conservar la biodiversidad en la Amazonia brasileña. También los estudios de Ehrlich y Pringle (2008) y Richardson y Loomis (2009) indican que las personas se inclinan a pagar por la conservación de especies carismáticas en muchos de los casos influenciadas por sus características estéticas. Sin embargo, estos autores reportan el poco conocimiento sobre el valor ecológico para otros animales como especies endémicas sin carisma, especies que no son ni endémicas ni carismáticas; así mismo indican que hace falta claridad sobre los factores que llevan al público a priorizar los tipos de especies a patrocinar.

Estudios en el ámbito educativo francés Ballouard, Provost, Barré y Bonnet (2012), al comparar contenidos de imágenes de internet, encontraron que la niñez en edad escolar tenía mayor predilección por animales exóticos que no hacen parte de la fauna local; este estudio sugiere implementar políticas educativas hacia el conocimiento de la biodiversidad local.

En consonancia con lo anterior, la transformación que han tenido los ecosistemas en los últimos tiempos ha repercutido en la disminución significativa de muchas especies y en crear conciencia de que el planeta se encuentra en medio de una crisis (Morse-Jones et al., 2012). Al respecto, Dunn (2005) indica que los medios de comunicación y las organizaciones se centran en divulgar y promover estrategias de conservación de mamíferos y aves, olvidando el papel funcional de otras especies en los ecosistemas; por ende, es importante involucrar al estudiantado desde edades tempranas en el reconocimiento del papel ecológico de todas las especies, para que establezcan vínculos positivos con el ambiente. 
doi: http://dx.doi.org/10.15359/ree.22-3.4

URL: http://www.una.ac.cr/educare

CORREO: educare@una.cr

En este sentido, distintos aspectos pueden influir sobre la percepción en el momento de establecer estrategias de conservación de especies particulares. Por ejemplo, es sustancial valorar en qué medida los medios de comunicación influyen en las percepciones del estudiantado. Al respecto, Samways (1993) indica que el desprecio hacia los insectos es impedimento para su conservación. También Prokop y Fančovičová (2010) analizan cómo el miedo puede influir fuertemente sobre el carisma hacia grandes carnívoros y serpientes: en su estudio, muestra el acuerdo de la niñez en la reducción de poblaciones de osos.

Estudios de Kellert (1984) y Ballouard et al. (2012) indican que el público en general solo reconoce unos pocos animales silvestres en comparación con las especies carismáticas, algunas de ellas exóticas y mascotas; aspecto que es preocupante para la conservación, porque descarta la opción de desarrollar iniciativas de participación de la ciudadanía para la conservación de especies locales. Por ende, dichas investigaciones sugieren que una de las maneras eficientes de corregir este sesgo es actualizar la educación biológica y ambiental de la niñez.

Las consideraciones anteriores conducen a la realización de este estudio, el cual pretende describir los efectos que tienen algunos medios de comunicación sobre el conocimiento de 20 especies de fauna; de igual forma se valora el impacto que tienen las imágenes en el direccionamiento de preferencias de la niñez hacia 10 especies de fauna. El estudio compara dos entornos educativos: rural y urbano.

Esta iniciativa puede dar paso a estudios posteriores para ampliar caracterizaciones sobre las concepciones del estudiantado; por ejemplo, Bell (1981) encontró que solo el 50\% de la niñez sabe que las ranas son anfibios. Estudios de Braund (1998) y Yen, Yao, y Chiu (2004) reportan otro tipo de concepciones, como considerar que la tortuga es un anfibio o clasificar la serpiente como invertebrado por la ausencia de extremidades. Las investigaciones de Braund (1998) y Trowbridge y Mintzes (1988) señalan que la niñez tiende a considerar el pingüino como un mamífero, porque no puede volar.

\section{Metodología}

Participantes: Los instrumentos fueron diligenciados por 249 estudiantes de cinco instituciones educativas, de las cuales tres son rurales y las otras son urbanas (Figura 1a). Dichas instituciones fueron elegidas por muestreo no probabilístico, considerando la voluntad de participación en el estudio. En el estudio participaron estudiantes de dos departamentos de Colombia (Boyacá y Santander); 124 estudiantes pertenecen a instituciones educativas rurales: 54 de Soracá (Rural A), 39 Ventaquemada (Rural B) y 31 Barbosa (Rural C) y, 125 estudiantes son de instituciones educativas urbanas de la ciudad de Tunja: 77 niñas y niños están matriculados en colegios de carácter público (y 48 son de colegios privados). La edad de las personas participantes oscila entre los 7 y los 13 años. El 53.8\% son mujeres y el $46.2 \%$ son hombres (Figura 1 b). 


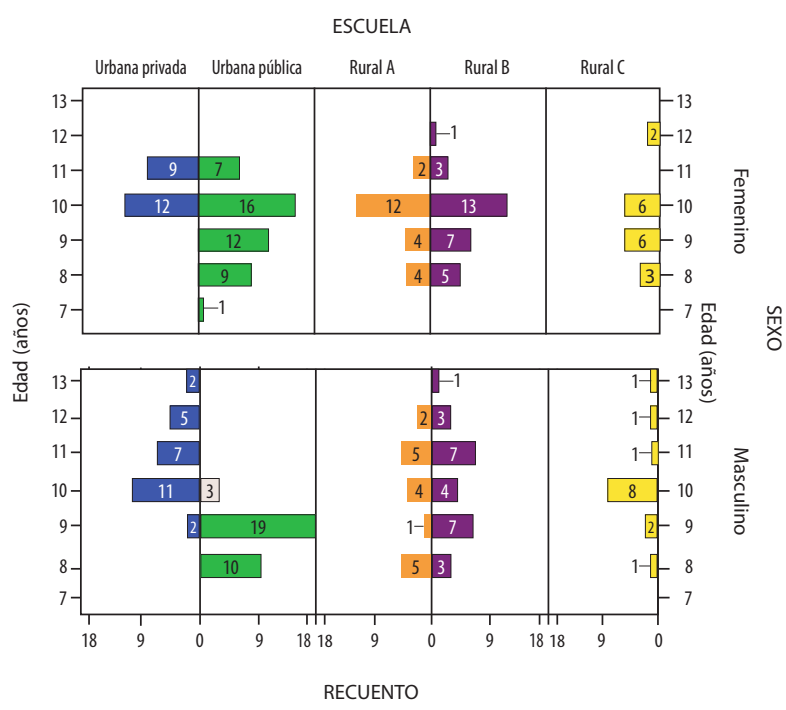

Figura 1a: Número de participantes por cada institución educativa. Nota: Elaboración propia.

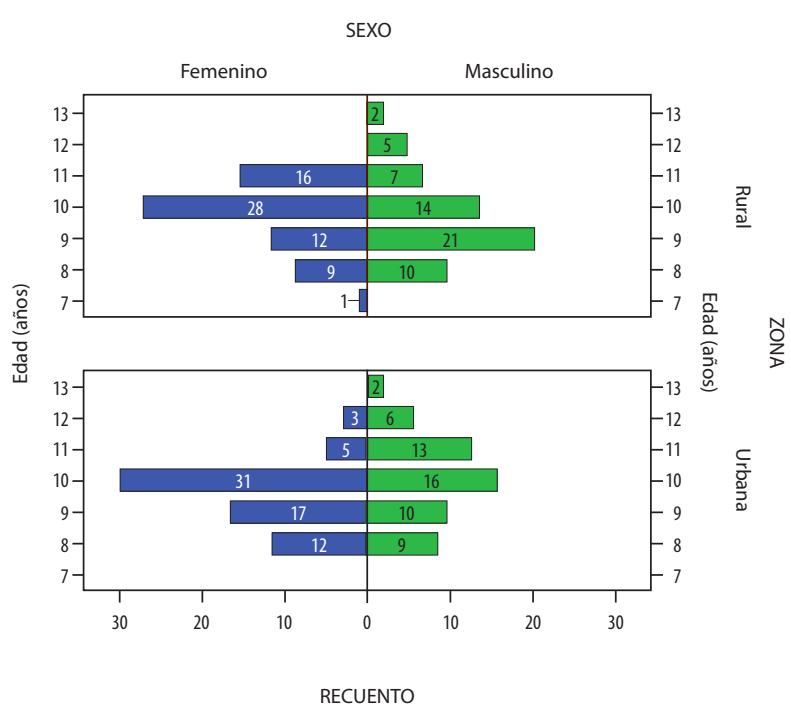

Figura 1b: Distribución por sexoy tipos de instituciones de participantes. Nota: Elaboración propia.

\section{Los instrumentos}

De acuerdo con investigaciones en educación acerca de percepciones sobre el conocimiento de la niñez sobre la biodiversidad, específicamente en lo relacionado con especies carismáticas y no carismáticas, Balmford, Clegg, Coulson y Taylor (2002), Snaddon, Turner y Foster (2008) han utilizado imágenes para identificar el grado de aprecio hacia la fauna. En este trabajo, se utilizaron dos tipos de presentaciones en PowerPoint:

- Una primera, presenta las imágenes de 4 especies por grupo taxonómico: mamíferos (zarigüeya, manatí, oso polar, mapache), reptiles (serpiente coral, camaleón, anaconda, tortuga), anfibios (rana azul, rana roja, rana de ojos rojos, salamandra), aves (copetón, colibrí, pelicano, cóndor) y artrópodos (mariposa, tarántula, escarabajo hércules, escarabajo estercolero). En función de esta presentación se pregunta al estudiantado dónde había visto el animal. El estudiantado registra sus respuestas en una hoja según las siguientes opciones: revistas o libros, televisión, internet, directamente en la naturaleza, no lo ha visto.

- En la segunda, se presentan dos imágenes por cada grupo taxonómico: mamíferos (manatí, zarigüeya), reptiles (serpiente coral, camaleón), anfibios (salamandra, rana de ojos rojos), aves (cóndor, colibrí) y artrópodos (tarántula, mariposa). El estudiantado indica, de manera escrita, en una matriz, si era agradable o desagradable 5 .

\footnotetext{
${ }^{5}$ Para el caso del estudiantado más pequeño se grabaron en video las sesiones con el fin de confirmar sus respuestas.
} 
doi: http://dx.doi.org/10.15359/ree.22-3.4

URL: http://www.una.ac.cr/educare

CORREO: educare@una.cr

La primera parte tiene como objetivo identificar cuáles son los medios de comunicación que tienen mayorincidenciaen la divulgación del conocimiento deespecies carismáticasynocarismáticas. La segunda parte pretende identificar cuáles son las especies más agradables o desagradables. Las imágenes fueron elegidas de manera que incluyeran animales exóticos y silvestres.

La aplicación del instrumento se realizó durante los meses de octubre 2013 a mayo de 2014. La toma de datos se efectuó en condiciones normales de clase, con la colaboración de dos estudiantes del grupo de investigación WAIRA (ambiente, comunidad y desarrollo) de la Universidad Pedagógica y Tecnológica de Colombia (UPTC), para realizar las grabaciones de video y audio en todas las sesiones. La docente titular estuvo presente en el desarrollo de las actividades.

Las respuestas obtenidas fueron consideradas de acuerdo con la opción elegida por el estudiantado, se mide la frecuencia en cada una de las opciones. Con estos datos se pudo valorar la influencia de los medios de comunicación e inferir aspectos emocionales hacia cada una de las especies presentadas.

\section{Resultados y análisis}

\section{Influencia de medios de comunicación en la identificación de grupos taxonómicos}

Los resultados muestran que la televisión (TV) y el internet (Figura 2a y b) son los medios de comunicación más señalados por el estudiantado en relación con la identificación de grupos taxonómicos como mamíferos y reptiles y en menor proporción los anfibios y artrópodos. Por ejemplo, se identifica que especies como el oso polar son vistas en la TV en un $73,9 \%$, en comparación con especie como la zarigüeya que son vistas en un 57\%. En el caso del internet, el animal que más ha sido visto por este medio es el camaleón con un 15,7\% y en menores porcentajes (3\%) se encuentra la mariposa y tarántula.

Estos resultados son similares a los de Campos (2012) e indican que una fuente de divulgación en fauna y flora es la TV. De la misma forma la diferencia de \% entre el oso polar y la zarigüeya permite coincidir con los resultados de Ballouard et al. (2012) sobre las preferencias hacia especies de fauna que no son necesariamente locales.

En relación con la opción "directamente en la naturaleza" (Figura 2c), el animal que más ha sido observado en la naturaleza es la mariposa con un $83,9 \%$, seguido de especies de aves como el copetón y colibrí, estas por ser especies con las que se tiene contacto cotidianamente. Especies como la zarigüeya, el manatí y el oso polar presentan porcentajes inferiores al 9\%, las dos últimas son animales carismáticos, lo cual no implica que hayan sido vistos directamente en su medio ambiente, en su defecto fueron vistos en medios naturales; pero a través de un medio de comunicación. 
doi: http://dx.doi.org/10.15359/ree.22-3.4

URL: http://www.una.ac.cr/educare

CORREO: educare@una.cr

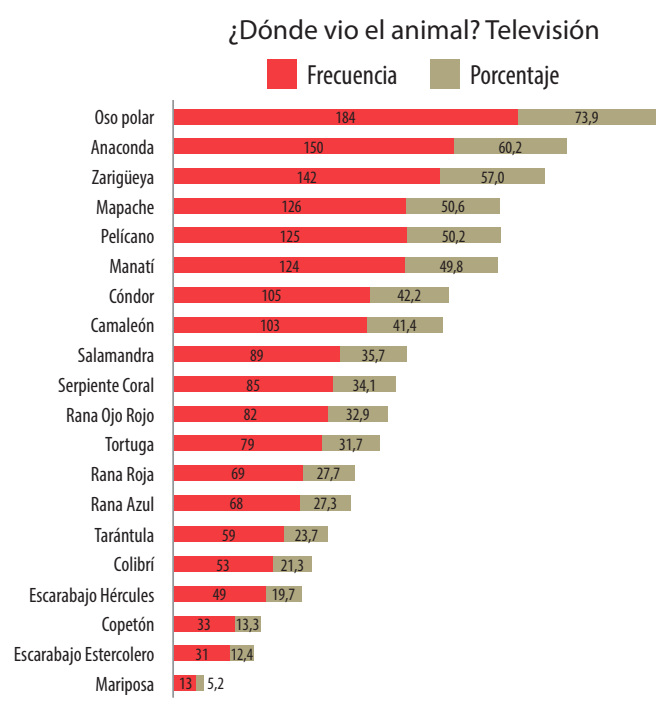

Figura 2a: \% y Frecuencias en la opción TV. Nota: Elaboración propia.

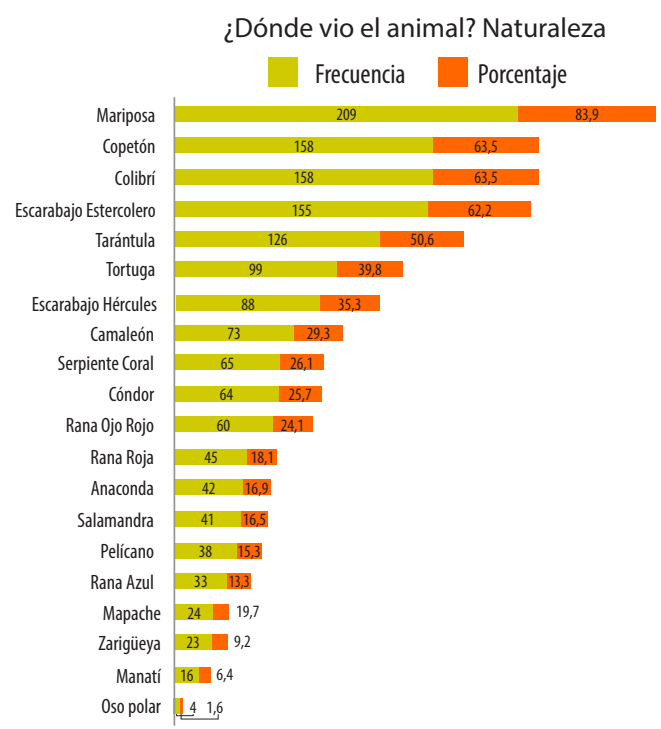

Figura 2c: \% y Frecuencias en la opción naturaleza. Nota: Elaboración propia.

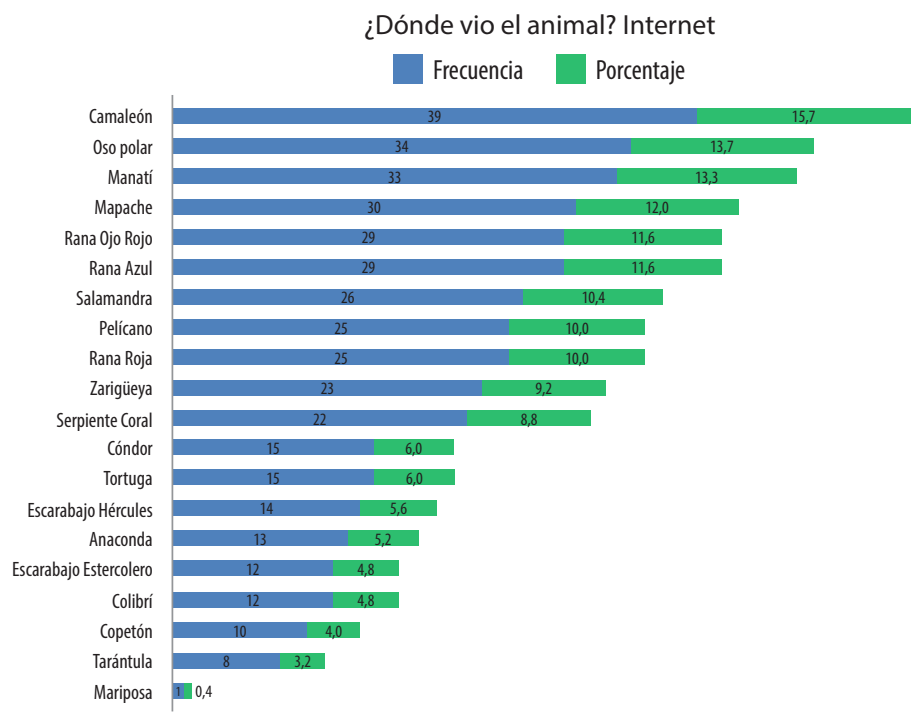

Figura $\mathbf{2}$ b: \% y Frecuencias en la opción internet.

Nota: Elaboración propia.

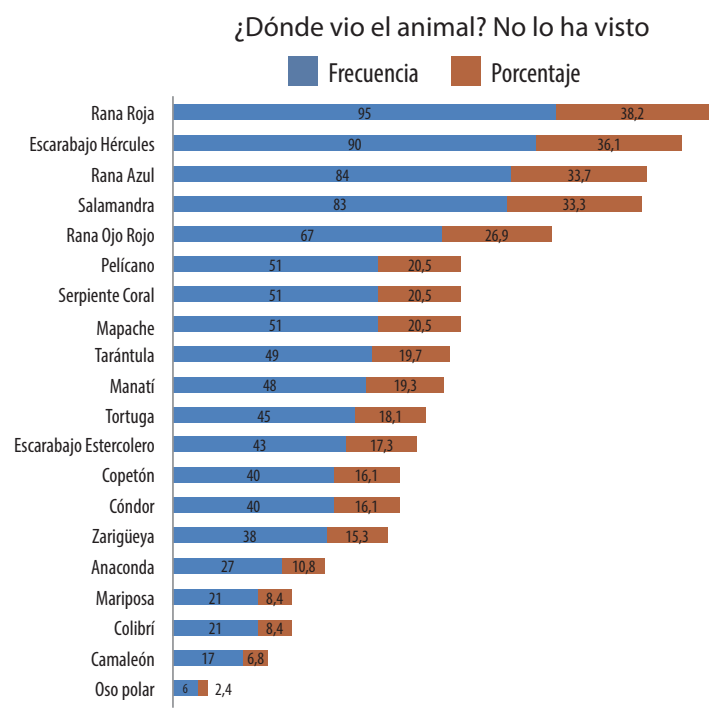

Figura 2d: \% y Frecuencias en la opción no lo ha visto.

Nota: Elaboración propia.

Esto permite tener en consideración, como aspecto educativo, el promover las salidas de campo en los planes curriculares, para dar la oportunidad al estudiantado de desarrollar actividades en la naturaleza y en especial de suscitar actitudes de conocimiento y respeto hacia la 
doi: http://dx.doi.org/10.15359/ree.22-3.4

URL: http://www.una.ac.cr/educare

CORREO: educare@una.cr

biodiversidad. Así mismo, Sudarmadi et al. (2001) destacan la necesidad de promover programas educativos que proporcionen herramientas para que las personas puedan reconocer los valores y aclarar conceptos, con el fin de desarrollar actitudes para comprender la interrelación entre los seres humanos y el entorno biofísico.

Investigaciones de Ballouard et al.(2012) sobre cambio de percepciones hacia las serpientes con niños y niñas afirman que el contacto físico con los animales parece ser el elemento crucial para mejorar la actitud de escolares hacia un organismo impopular.

En la opción "no ha visto el animal" y de acuerdo con la Figura 2d, el estudiantado señala especies como rana roja $38 \%$, el escarabajo hércules $36 \%$ y el oso polar en un $2,4 \%$. Estos datos muestran cómo el estudiantado tiene mayor conocimiento de especies exóticas como el oso polar en relación con especies que hacen parte de la fauna latinoamericana.

\section{Comparación entre colegios rurales y urbanos}

Al valorar algunas diferencias entre colegios rurales y urbanos, se observa que el estudiantado urbano indica con mayor frecuencia la opción "internet" como fuente de conocimiento de especies -camaleón, oso polar, manatí y mapache- (Figura 3a). En la opción TV, este estudiantado elige más esta opción para especies como escarabajo hércules, rana azul, rana de ojos rojos, salamandra (Figura 3d). También tienden a señalar la opción directamente en la naturaleza en animales como manatí, mapache, salamandra, rana de ojos rojos (Figura $3 b)$. Es de aclarar que estos fueron vistos en ambientes como zoológicos, acuarios que son simulaciones de ambientes naturales. El estudiantado urbano señala en mayor frecuencia la opción revistas y libros, este aspecto puede ser explicado por la diversidad de libros de texto en la biblioteca de estas instituciones (Ver Figura 3c).

El estudiantado rural señala con mayor frecuencia la opción "no ha visto el animal", en especies como anaconda, mapache, manatí, serpiente coral, salamandra y rana roja. En especies como camaleón, tortuga, escarabajo estercolero, la opción "directamente en la naturaleza" fue mayor, este aspecto nuevamente permite destacar el aprovechamiento de las salidas de campo, como opción de conocimiento de la biodiversidad en la escuela. La opción TV fue más señalada para especies como oso polar y anaconda, por ser especies exóticas.

Analizando el tipo de elección por grupo taxonómico se observa que, en los mamíferos, el estudiantado urbano, además de la TV, señala otras fuentes de información como las revistas, el internet; mientras que el estudiantado rural, en su mayoría, solo señala la televisión.

En relación con reptiles, el estudiantado urbano indica en mayor frecuencia la opción directamente en la naturaleza para la anaconda, esto puede ser explicado porque gran cantidad de estudiantes tienden a confundir ambientes como el zoológico con ambientes naturales. 


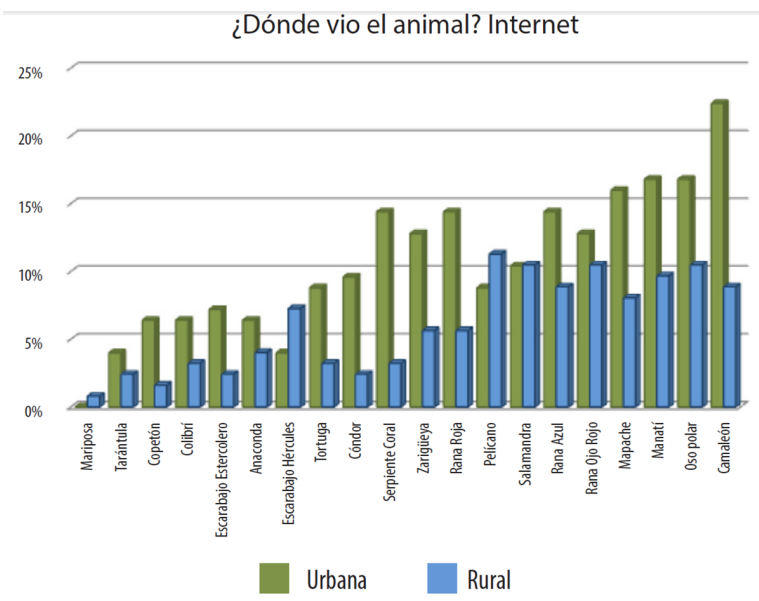

Figura 3a: Fuente de conocimiento internet. Nota: Elaboración propia.

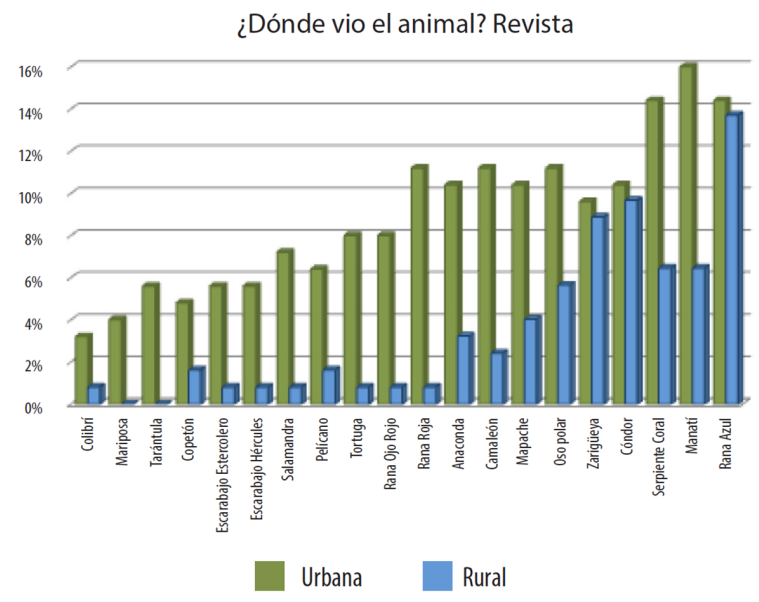

Figura 3c: Fuente de conocimiento revista. Nota: Elaboración propia.

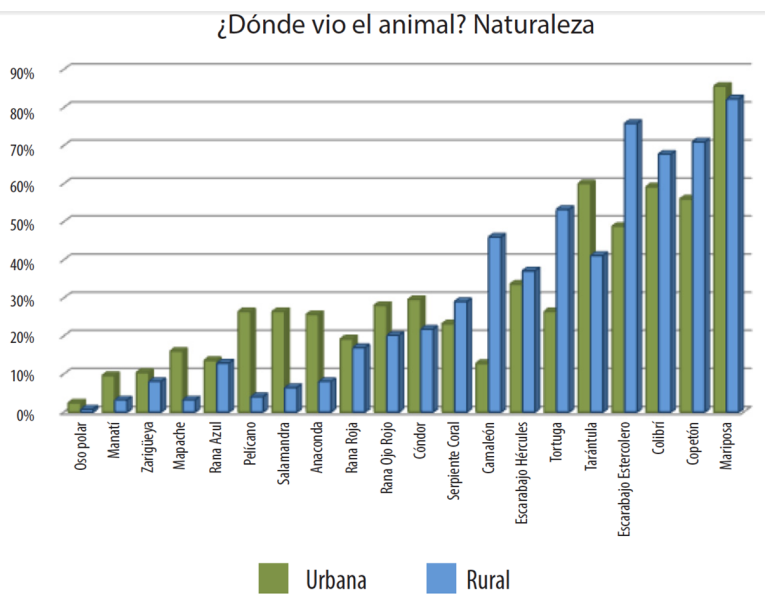

Figura 3b: Fuente de conocimiento naturaleza. Nota: Elaboración propia.

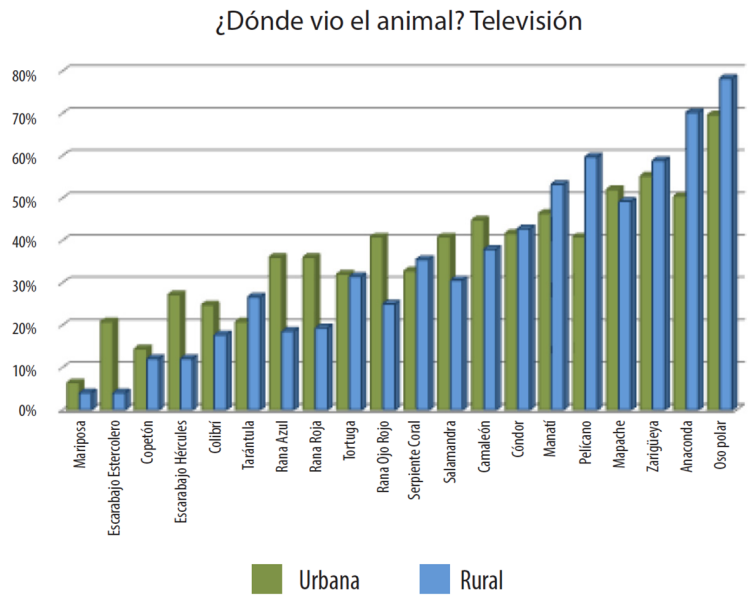

Figura 3d: Fuente de conocimiento televisión. Nota: Elaboración propia.

En el grupo de los anfibios se observan porcentajes mayores en la opción no ha visto el animal, en entornos rurales. En el caso de las aves, especies como colibrí y copetón, por ser especies endémicas, el estudiantado señala en un $63,5 \%$ observarlas directamente en la naturaleza, caso contrario al del pelicano y cóndor que son identificado por medios como la TV y el internet. Frente a los artrópodos en especies como el escarabajo estercolero, mariposa, tarántula, el estudiantado rural y urbano elige, de manera similar, la opción directamente en la naturaleza.

Los resultados permiten indicar que el estudiantado tiene mayor conocimiento de especies de mamíferos, reptiles y aves, por medios de comunicación como la TV, el internet o 
doi: http://dx.doi.org/10.15359/ree.22-3.4

URL: http://www.una.ac.cr/educare

CORREO: educare@una.cr

por verlos directamente en la naturaleza. Esto no sucede con especies de anfibios y artrópodos. Se observa que la niñez rural y urbana está más habituada a especies exóticas que a especies endémicas por conocimiento de fuentes como TV, internet; pues, en la mayoría de casos, las campañas publicitarias de ONG se centran en la megafauna, fauna silvestre, específicamente mamíferos, como lo reportan los trabajos de Campos (2012), Paraskevopoulos, Padeliadu y Zafiropoulos (1998), Lock (1995) y Tisdell (2011).

\section{Percepciones sobre algunos grupos taxonómicos}

Para describir los resultados, producto de la segunda presentación en Power-point, que permite valorar si las especies que se mostraban son agradables o desagradables, en primer lugar se hace una descripción teniendo en cuenta el género, luego de acuerdo con el tipo de institución y, por último, se considera la edad.

El género masculino y femenino tiene predilección por aves y mamíferos. Los niños indican más agrado hacia los reptiles, artrópodos y los anfibios que las niñas. El género femenino parece familiarizarse con las especies exóticas. Esto mismo fue encontrado por Snaddon et al. (2008) en su trabajo sobre percepciones de la niñez acerca de la biodiversidad selvática en el Reino Unido. También en el estudio de Campos (2012) se indica que los niños mencionan más insectos, reptiles y anfibios que las niñas (Figura, 4a).

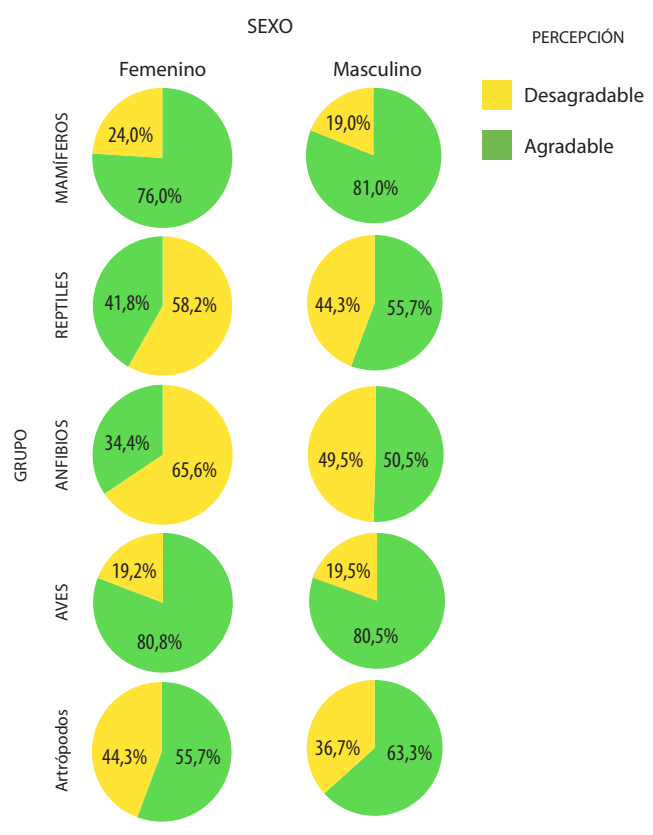

Figura 4a: Preferencia por género según grupo taxonómico. Nota: Elaboración propia.

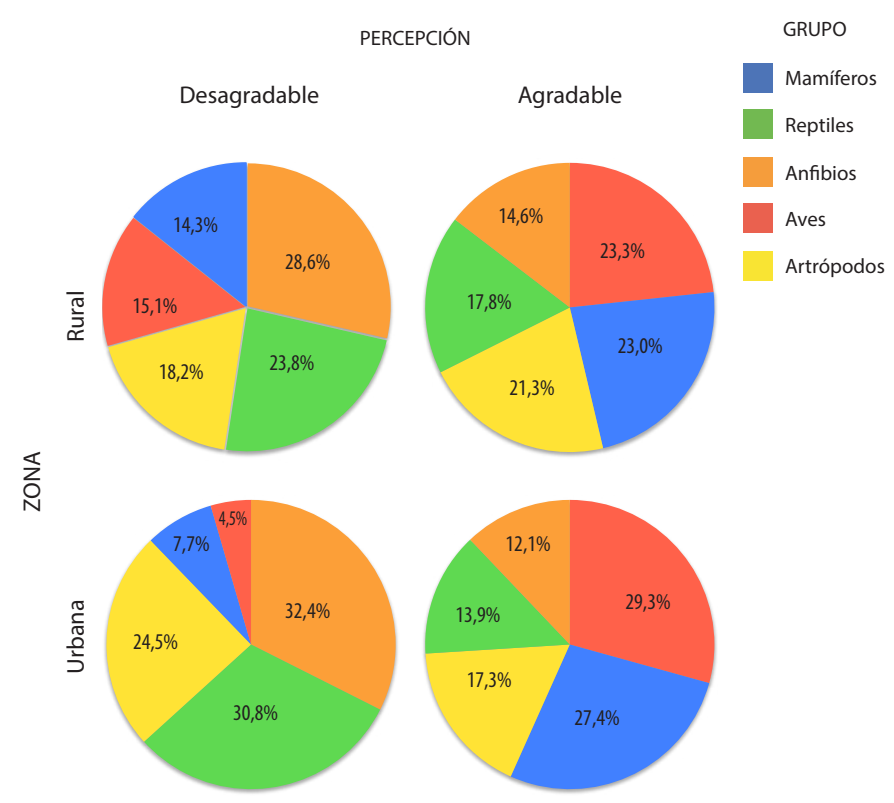

Figura 4b: Preferencias por tipo de institución (rural, urbana) según grupo taxonómico. Nota: Elaboración propia. 
En la Figura $4 \mathrm{~b}$ se observan las diferencias entre instituciones rurales y urbanas. El estudiantado del contexto urbano tiende a considerar más agradables a los mamíferos, los reptiles, artrópodos y los anfibios tienden a ser más agradables en la niñez del contexto rural. La preferencia hacia los mamíferos coincide con estudios de Campos (2012), Lindemann-Matthies (2006), Snaddon et al. (2008), quienes indican que esto se debe a características antropomorfas, por ser grandes y carismáticos. En el estudio realizado por Campos (2012) se encuentra que el efecto de procedencia de la niñez (rural o urbana) no es directo; empero, en relación con el género, los niños rurales tienen más conocimiento de las especies nativas. Así mismo, la autora hace énfasis en la salida de campo como principal fuente de contacto con la biodiversidad.

Para analizar si las edades influyen en el tipo de percepción del estudiantado en relación con las distintas especies de los grupos taxonómicos, se hace uso de la Figura 5, en la cual la parte izquierda de cada figura hace referencia a aquellas especies que son consideradas desagradables por el grupo de estudiantes participantes en el estudio y, la parte derecha corresponde a la opción agradable. Así, la serpiente coral y la tarántula tienden a mantenerse en la parte izquierda de las figuras en las distintas edades indicando que son desagradables.
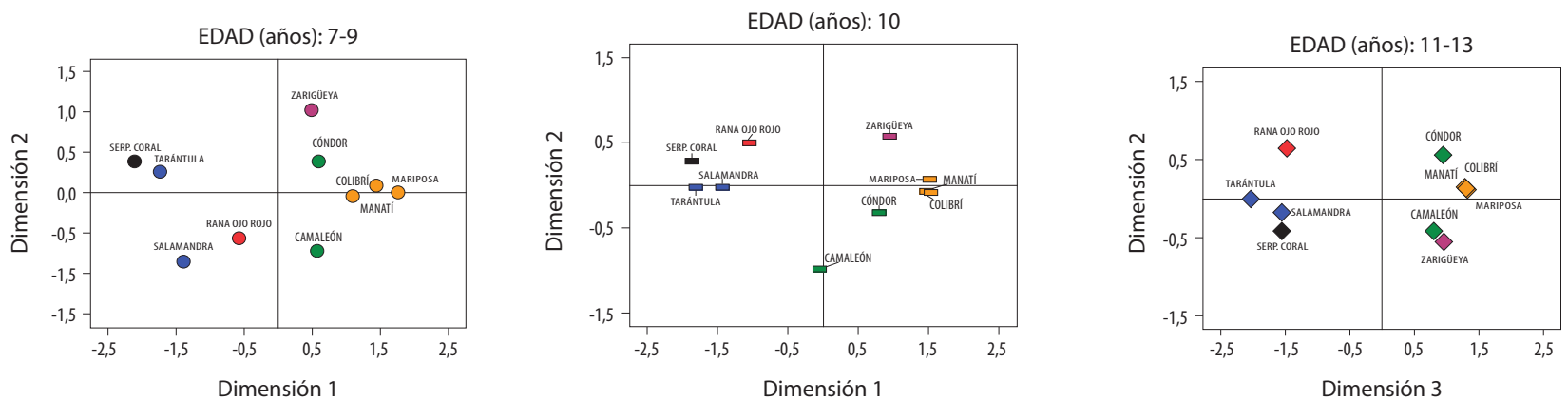

Figura 5: Preferencias por edades hacia distintas especies de fauna. Nota: Elaboración propia.

Se identifica que, a medida que aumenta la edad, el porcentaje de la opción "agradable" se incrementa para mamíferos, reptiles y aves. Para anfibios y artrópodos la opción "desagradable" se incrementa levemente a medida que aumenta la edad. Algunas especies como el colibrí, el camaleón, el manatí, la mariposa tienden a permanecer iguales en edades de 7 a 10 años y de 11 a 13 años (Figura 5).

Por ejemplo, en estudiantes con edades inferiores a 10 años, se considera la rana de ojos rojos y la salamandra como desagradables, estas apreciaciones parecen cambiar en edades superiores donde se muestra que son agradables. 
doi: http://dx.doi.org/10.15359/ree.22-3.4

URL: http://www.una.ac.cr/educare

CORREO: educare@una.cr

Ahora, teniendo en cuenta las especies, las más desagradables son: tarántula, serpiente coral, salamandra y rana de ojos rojos. Se observa que estos valores tienden a ser mayores en los contextos urbanos. Especies como el cóndor, el manatí, el colibrí tienen porcentajes más altos en la opción "desagradable" en el contexto rural. Para estudiantes rurales y urbanos, hay similitud en el agrado de especies como la mariposa (Figura 6a).

El estudio de Ballouard et al. (2012) señala que, si se realiza un trabajo educativo con el estudiantado sobre las serpientes, este puede mostrar un cambio de actitud frente a estas. Los autores sugieren promover salidas de campo que permitan aumentar el conocimiento ecológico acerca de especies silvestres.

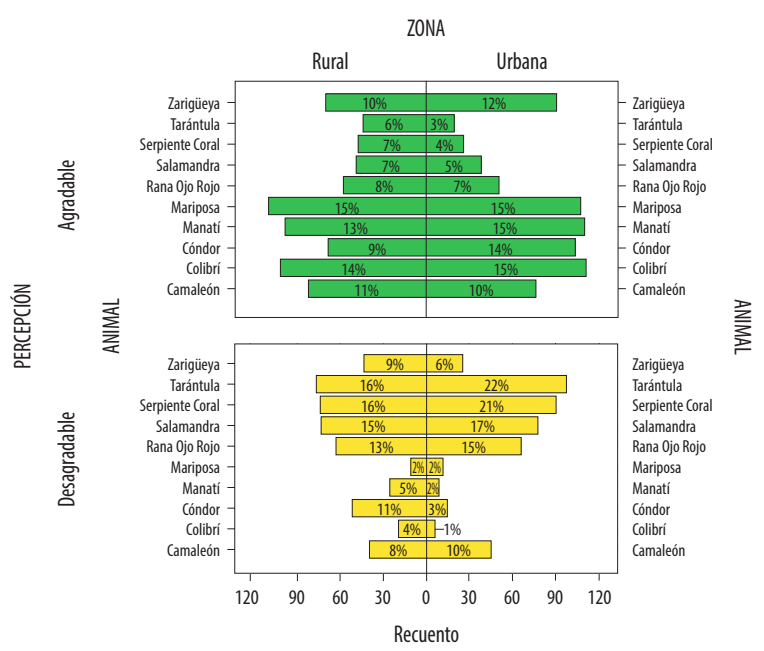

Figura 6a: Preferencia por tipo de institución (rural, urbana) en relación con las distintas especies.

Nota: Elaboración propia.

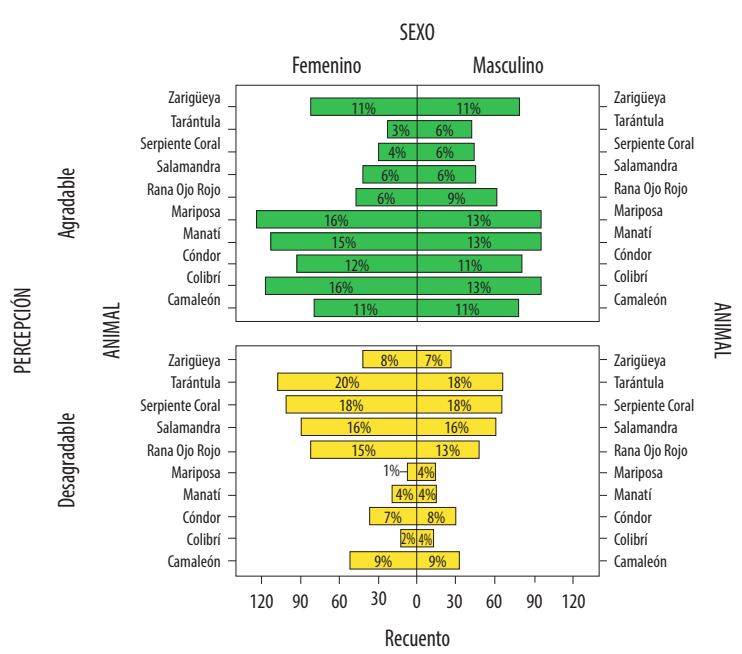

Figura 6b: Preferencia por género (Masculino, femenino) en relación con las distintas especies.

Nota: Elaboración propia.

Las preferencias están relacionadas con las características antropocéntricas y antropomórficas en cuanto al lugar (Kellert, 1984, 1993; Meuser, Harshaw, Mooers, 2009; Tisdell, 2011). Un ejemplo claro está en los mamíferos que se mostraron en las diapositivas; mientras para la niñez rural y urbana la zarigüeya solo alcanza el 10 y $12 \%$ en la opción agradable, el manatí alcanza el 13 y $15 \%$. Caso contrario sucede con la tarántula, donde solo el $6 \%$ y $3 \%$ en entornos rurales y urbanos señala que son agradables. En relación con las aves, el cóndor tiende a ser más desagradable para el estudiantado rural (11\%) que para los urbanos en un $3 \%$; mientras que en el colibrí se observan porcentajes mayores en la opción agradable.

En la Figura $6 b$, se muestra que las niñas tienden a mayor preferencia por especies de colores llamativos como el colibrí, camaleón y la mariposa. En el caso de los niños estos tienden a mostrar mayor agrado en especies como serpiente coral, tarántula y rana de ojos rojos. 
Se evidencia que especies como manatí, colibrí y mariposa tienden a ser consideradas muy agradables, en un $88,6 \%$, otras especies como zarigüeya, camaleón y cóndor también tienden a ser consideradas agradables, pero con un porcentaje menor 69,2\%. Caso contrario, en grupos de especies como la serpiente y la tarántula, el porcentaje de desagradable llega hasta el 71,1\% (Figura 7a).

Con el fin de evaluar el grado de asociación que existe entre las variables especie y percepción, se calculó el valor del estadístico V de Cramer. Para ello se tomaron cada una de las posibles parejas de especies y las dos categorías de la variable percepción (agradable y desagradable). Este tipo de asociaciones puede detallarse en la Figura 7b; por ejemplo, se observa que mariposa y tarántula presentan una asociación moderada (0.64) frente a ser agradable o desagradable; para este caso, mientras a un estudiante le agrada la mariposa, le desagrada la tarántula. De otra parte, el valor de 0.04, para el caso de mariposa y colibrí, significa que existe muy poca asociación de estas especies con las categorías de agradable y desagradable; sin embargo, de acuerdo con la Figura 5 se puede establecer que, a la mayoría de estudiantes, le agrada la mariposa y también le agrada el colibrí. Una interpretación similar se puede hacer para el valor 0.05 (serpiente coral y tarántula); pero, en este caso, a la mayoría de estudiantes le desagrada este tipo de especies

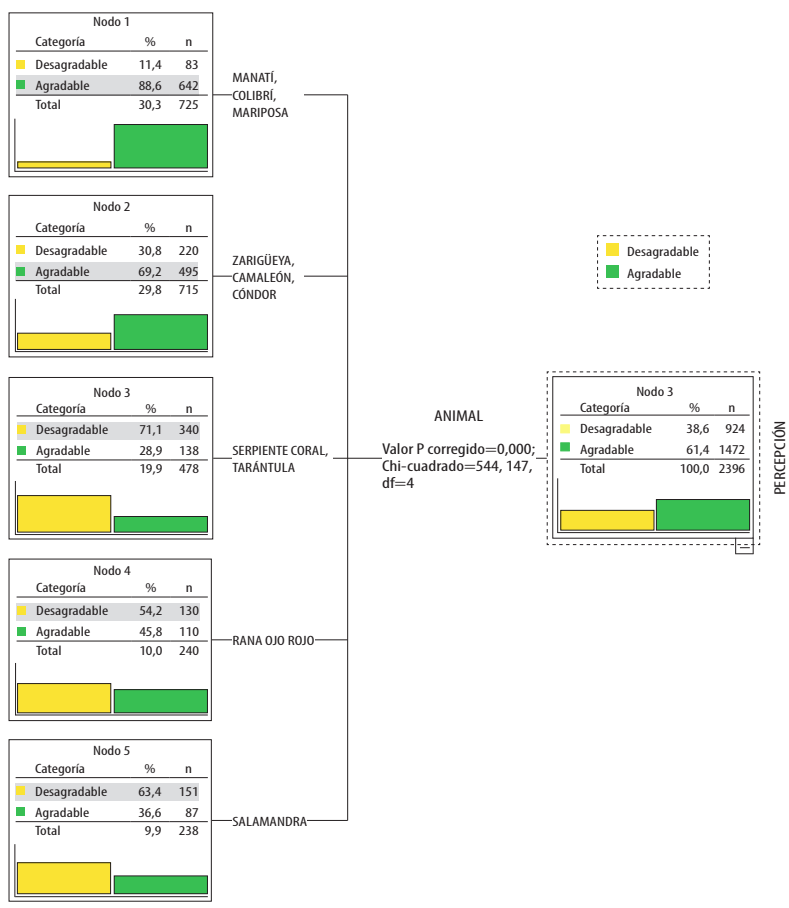

Figura 7a: Porcentajes de especies agradables y desagradables. Nota: Elaboración propia.

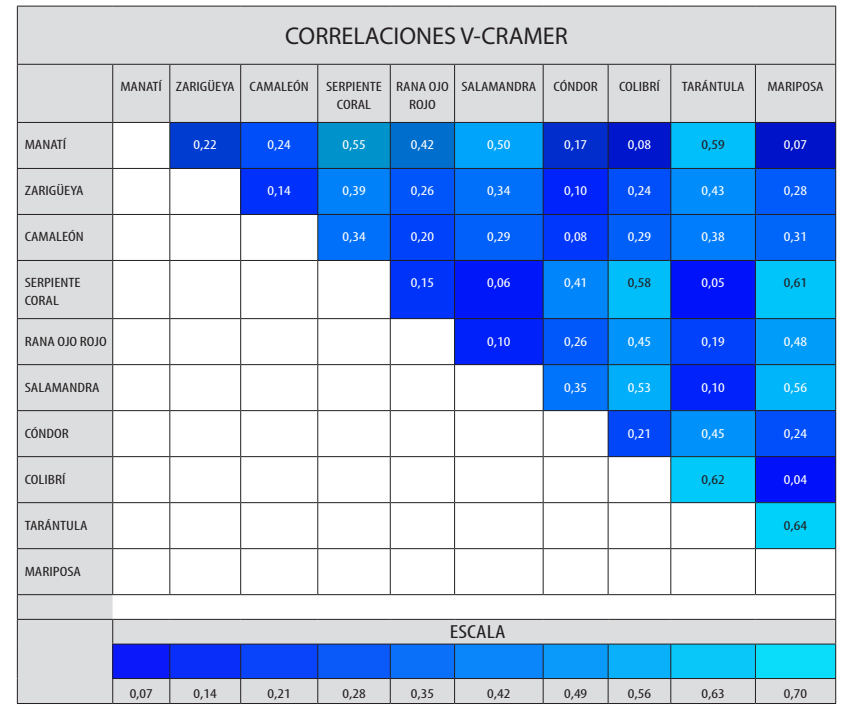

Figura 7b: V-Cramer Grado de asociación entre especies de fauna. Nota: Elaboración propia. 
doi: http://dx.doi.org/10.15359/ree.22-3.4

URL: http://www.una.ac.cr/educare

CORREO: educare@una.cr

\section{Conclusiones e implicaciones didácticas}

Los resultados muestran la gran influencia que ejercen los medios como la TV y el internet, por lo tanto, es un punto de partida que permite valorar cómo se pueden utilizar estas experiencias para favorecer el conocimiento de la biodiversidad, junto con el papel biológico de cada especie en particular. Como lo señala Cebrián (1997), los medios de comunicación pueden transportar la realidad social y científica al aula y, en ocasiones, resultan ser más eficaces que el propio personal docente. Esto significa que pueden ayudar a contextualizar los procesos educativos. En general, los estudiantes y las estudiantes participantes indican que tienen conocimiento, por medios como la TV, de la anaconda, camaleón y serpiente.

Lo anterior permite plantearnos cuestiones que hacen énfasis en los mensajes publicitarios sobre la biodiversidad y su importancia de trabajar estos contenidos con el estudiantado, para el desarrollo de su capacidad crítica, acompañado de salidas de campo donde el estudiantado pueda efectuar diversas actividades en la naturaleza. Por ende, los medios de comunicación deberían incluir, con mayor grado de equidad, el estudio de biodiversidad específicamente sobre la fauna endémica y no carismática, esto favorecería su conocimiento y reconocimientos acerca de las funciones biológicas que estas especies representan.

Al parecer la niñez conoce más sobre especies exóticas que nativas y tiene una particular atención por especies de mamíferos. Esto es trascendente, porque las actitudes de las personas hacia las especies definen, en gran parte, el comportamiento que tendrán hacia la biodiversidad (Campos, 2012; Pooley y O'Connor, 2000).

En el caso de artrópodos como la tarántula, serpiente coral, a pesar de su importancia ecológica, estos animales despiertan aversión y miedo (Dunn, 2005). El estudio planteado permite estar de acuerdo con Ballouard et al. (2012) y Miller (2005) quienes enfatizan que la falta de conocimiento sobre el papel ecológico, acompañada de procesos de educación ambiental, afectan de forma negativa el apoyo del público hacia la conservación de la biodiversidad y hacen énfasis en cómo las salidas de campo, al incluir el contacto físico con la vida silvestre en los sistemas educativos, promueven un cambio de actitud hacia especies que en general despiertan miedo, como las serpientes. Por tanto, la existencia y el futuro de la biodiversidad dependen tanto de los procesos biológicos como educativos (Lindemann-Matthies, 2006; Torres y Medina, 2014).

Así mismo, se recomienda analizar la pertinencia y utilidad de los materiales utilizados en la enseñanza de temas como la biodiversidad en particular sobre las especies no carismáticas, de manera que el personal docente diseñe estrategias pedagógicas que dinamicen su enseñanza.

Se sugiere abordar el tema de biodiversidad de manera específica en los lineamientos curriculares de la educación primaria, en particular, sobre especies no carismáticas, pues esto contribuiría a desarrollar puntos de vista más informados acerca de la función biológica sobre estas especies en un ecosistema. 


\section{Agradecimientos}

A la Dirección de investigaciones (DIN) de la Universidad Pedagógica y Tecnológica de Colombia por financiar el proyecto denominado: Evaluación de la popularidad de especies carismáticas y no carismáticas en niños de primaria. Contribuciones a la educación ambiental para la biodiversidad (Torres-Merchán, 2013); así como a las instituciones educativas, estudiantes y profesorado participantes.

\section{Conflicto de intereses}

El presente manuscrito no presenta conflicto de intereses porque es el resultado de un proyecto de investigación ejecutado por el equipo responsable de este artículo y, por lo tanto, declaramos que es un producto original.

\section{Referencias}

Ballouard, J.-M., Provost, G., Barré, D. y Bonnet, X. (2012). Influence of a field trip on the attitude of schoolchildren toward unpopular organisms: An experience with snakes. Journal of Herpetology, 46(3), 423-428. doi: https://doi.org/10.1670/11-118

Balmford, A., Clegg, L., Coulson, T. y Taylor, J. (2002). Why conservationists should heed Pokémon. Science, 295(5564), 2367-2367. doi: https://doi.org/10.1126/science.295.5564.2367b

Bell, B. F. (1981). When is an animal, not an animal? Journal of Biological Education, 15(3), 213218. doi: https://doi.org/10.1080/00219266.1981.9654381

Braund, M. (1998). Trends in children's concepts of vertebrate and invertebrate. Journal of Biological Education, 32(2), 112-118. doi: https://doi.org/10.1080/00219266.1998.9655606

Campos, C. (2012). Los niños y la biodiversidad. ¿Qué especies conocen y cuáles son las fuentes de conocimiento sobre la biodiversidad que utilizan los estudiantes? Un aporte para definir estrategias educativas. Boletín Biológica, 24(6), 4-9.

Cebrián, M. (1997). ¿Qué y cómo aprenden los niños y las niñas desde la televisión? En J. I. Aguaded (Dirección), La otra mirada a la tele. Pistas para un consumo inteligente de la televisión (pp. 87-96). España: Grupo Comunicar.

Dunn, R. (2005). Modern insect extinctions, the neglected majority. Conservation Biology, 19(4), 1030-1036. doi: https://doi.org/10.1111/j.1523-1739.2005.00078.x

Ehrlich, P. R. y Pringle, R. M. (2008). Where does biodiversity go from here? A grim businessas-usual forecast and a hopeful portfolio of partial solutions. Proceeding of the National Academy of Sciences. 105(Supplement 1), 11579-11586. doi: https://doi.org/10.1073/ pnas.0801911105 
doi: http://dx.doi.org/10.15359/ree.22-3.4

URL: http://www.una.ac.cr/educare

CORREO: educare@una.cr

Horton, B., Colarullo, G., Bateman, I. J. y Peres, C. A. (2003). Evaluating non-user willingness to pay for a large-scale conservation programme in Amazonia: A UK/Italian contingent valuation study. Environmental Conservation, 30(02), 139-146. doi: https://doi.org/10.1017/ $\underline{\text { S0376892903000122 }}$

Kellert, S. R. (1984). American attitudes toward and knowledge of animals: An update. En M. W. Fox y L. D. Mickley (Eds.), Advances in Animal Welfare Science (pp. 177-213). Dordrecht: Springer.

Kellert, S. R. (1993). The biological basis for human values of nature. En S. R. Kellert y E. O. Wilson (Eds.), The biophilia hypothesis (pp. 41-72). Washington: Island Press.

Lindemann-Matthies, P. (2006). Investigating nature on the way to school: Responses to an educational programme by teachers and their pupils. International Journal of Science Education, 28(8), 895-918. doi: https://doi.org/10.1080/10670560500438396

Lock, R. (1995). Biology and the environment-A changing perspective? Or 'there's wolves in them there woods!' Journal of Biological Education, 29(1), 3-4. doi: https://doi.org/10.1080 100219266.1995.9655409

Meuser, E., Harshaw, H. W. y Mooers, A. O. (2009). Public preference for endemism over other conservation-related species attributes. Conservation Biology, 23(4), 1041-1046. doi: https://doi.org/10.1111/j.1523-1739.2009.01257.x

Miller, J. R. (2005). Biodiversity conservation and the extinction of experience. Trends in ecology y evolution, 20(8), 430-434. doi: https://doi.org/10.1016/j.tree.2005.05.013

Morse-Jones, S., Bateman, I. J., Kontoleon, A., Ferrini, S., Burgess, N. D. y Turner, R. K. (2012). Stated preferences for tropical wildlife conservation amongst distant beneficiaries: charisma, endemism, scope and substitution effects. Ecological Economics, 78, 9-18. doi: https://doi. org/10.1016/j.ecolecon.2011.11.002

Paraskevopoulos, S., Padeliadu, S y Zafiropoulos, K. (1998). Environmental knowledge of elementary school students in Greece. The Journal of Environmental Education, 29(3), 5560. doi: https://doi.org/10.1080/00958969809599119

Pooley, J y O'Connor, M. (2000). Environmental education and attitudes: Emotions and beliefs are what is needed. Environment and behavior, 32(5), 711-723. doi: https://doi. org/10.1177/0013916500325007

Prokop, P y Fančovičová, J. (2010). Perceived body condition is associated with fear of a large carnivore predator in humans. Annales Zoologici Fennici, 47(6), 417-425. doi: https://doi. org/10.5735/086.047.0606 
Richardson, Ly Loomis, J. (2009). The total economic value of threatened, endangered and rare species: an updated meta-analysis. Ecological Economics, 68(5), 1535-1548. doi: https:// doi.org/10.1016/j.ecolecon.2008.10.016

Samways, M. J. (1993). Insects in biodiversity conservation: Some perspectives and directives. Biodiversity and conservation, 2(3), 258-282. doi: https://doi.org/10.1007/BF00056672

Snaddon, J. L., Turner, E. C., y Foster, W. A. (2008). Children's perceptions of rainforest biodiversity: Which animals have the lion's share of environmental awareness? PLoS One, 3(7), e2579. Doi: https://doi.org/10.1371/journal.pone.0002579

Sudarmadi, S., Suzuki, S., Kawada, T., Netti, H., Soemantri, S. y Tugaswati, A. T. (2001). A survey of perception, knowledge, awareness, and attitude in regard to environmental problems in a sample of two different social groups in Jakarta, Indonesia. Environment, development and sustainability, 3(2), 169-183. doi: https://doi.org/10.1023/A:1011633729185

Tarlowski, A. (2006). If it's an animal it has axons: Experience and culture in preschool children's reasoning about animates. Cognitive development, 21(3), 249-265. doi: https://doi. org/10.1016/j.cogdev.2006.02.001

Tisdell, C. (2011). Institutional economics and the behaviour of conservation organizations: Implications for biodiversity conservation. In K. N. Ninan (Ed.), Conserving and valuing ecosystem services and biodiversity. Economic, institutional and social challenges (pp. 175194). Earthscan, London.

Torres, N. Y. y Medina, N. A. (2014). Representaciones en estudiantes de básica primaria acerca de especies carismáticas y no carismáticas en instituciones educativas rurales y urbanas. Bio-grafia, 7(12), 21-32. doi: https://doi.org/10.17227/20271034.12biografia21.32

Torres-Merchán, N.Y.(2013). Evaluación de la popularidad de especies carismáticasyno carismáticas en niños de primaria. Contribuciones a la educación ambiental para la biodiversidad (Proyecto de Investigación, código SGI 1272). Colombia: Universidad Pedagógica y Tecnológica de Colombia.

Trowbridge, J.-E., y Mintzes, J. J. (1988). Alternative conceptions in animal classification: A cross-age study. Journal of Research in Science Teaching, 25(7), 547-571. doi: https://doi. org/10.1002/tea.3660250704

Yen, C. F., Yao, T.-W., y Chiu, Y. C. (2004). Alternative conceptions in animal classification Focusing on ampibians and reptiles: A cross-age study. International Journal of Science and Mathematics Education, 2(2), 159-174. doi: 10.1007/s10763-004 